\title{
Oxigenoterapia domiciliar prolongada: perfil dos usuários e custos
}

\author{
Long term home oxygen therapy: users' profile and costs \\ Oxigenoterapia domiciliaria prolongada: perfil de los usuarios y costos
}

\author{
Cristina Shizue WatanabeI; Leonardo Francisco Campos de AndradeII; Manoel Quintino da Silva NetoIII; \\ Silvia de Fátima Tamini dos Santos ${ }^{I V}$; Lauren Suemi Kawata ${ }^{V}$
}

\begin{abstract}
RESUMO: Estudo exploratório-descritivo, quantitativo, com objetivo de identificar e analisar o perfil dos usuários de oxigenoterapia domiciliar prolongada (ODP), demonstrando o custo mensal da ODP de tais usuários para o município. Foram utilizados dados secundários captados em prontuários, em julho de 2012. A população foi composta por 40 usuários de ODP cadastrados no Serviço de Atenção Domiciliar Norte de Ribeirão Preto-São Paulo. Entre os usuários, 67,5\% eram do sexo feminino; $30 \%$ tabagistas; $67,5 \%$ estavam na faixa de 60 anos ou mais; $62,5 \%$ tinham doença pulmonar obstrutiva crônica; $75 \%$ mostraram melhora nos níveis de saturação de oxigênio após inicio da ODP; $87,5 \%$ utilizavam cateter nasal e $50 \%$ usavam concentrador como fonte. O custo mensal somou $\mathrm{R} \$ 19.410,00$. Conclui-se que o perfil dos usuários reflete o envelhecimento da população e o predomínio de doenças crônicas relacionadas ao estilo de vida.
\end{abstract}

Palavras-Chave: Oxigenoterapia; assistência domiciliar; perfil de saúde; custos e análise de custos.

\begin{abstract}
Exploratory-descriptive, quantitative study aiming at identifying and analyzing the profile of users of long term home oxygen therapy (HOT), as well as identifying the monthly cost of the HOT for the city. Secondary data were obtained from medical records, in July, 2012. The population consisted of 40 users registered in HOT North Home Care in Ribeirão Preto, São Paulo, Brazil. Among the users, $67.5 \%$ were female; $30 \%$ were smokers; $67.5 \%$ were 60 years or older; $62.5 \%$ had chronic obstructive pulmonary disease; $75 \%$ showed improvement in oxygen saturation levels after initiation of HOT; $87.5 \%$ used nasal catheter, and $50 \%$ used concentrator as a source. Monthly cost was R $\$ 19,410.00$. Conclusions show that users' profile mirrors the ageing of the population and the prevalence of chronic diseases related to lifestyle.

Keywords: Oxygen inhalation therapy; home nursing; health profile; costs and costs analysis.
\end{abstract}

RESUMEN: Estudio exploratorio, descriptivo y cuantitativo, con el objeto de identificar y analizar el perfil de los usuarios de oxigenoterapia domiciliaria prolongada (ODP), señalando el costo mensual de la ODP de tales usuarios para el municipio. Se emplearon datos secundarios reflejados en historiales, en julio de 2012. El universo lo constituían 40 usuarios de ODP registrados en el Servicio de Atención Domiciliaria Norte de Ribeirão Preto-São Paulo. Entre los usuarios, el 67,5\% eran del sexo femenino; $30 \%$ tabaquistas; $67,5 \%$ tenían 60 años o más; $62,5 \%$ tenían enfermedad pulmonar obstructiva crónica; $75 \%$ reflejaron mejora en los niveles de saturación de oxígeno tras el inicio de la ODP; $87,5 \%$ empleaban catéter nasal y $50 \%$ empleaban concentrador como fuente. El costo mensual alcanzó $\mathrm{R} \$ 19.410,00$. Se concluye que el perfil de los usuarios refleja el envejecimiento de la población y el predominio de las enfermedades crónicas relacionadas con el modo de vida. Palabras Clave: Oxigenoterapia; asistencia domiciliaria; perfil de salud; costos y análisis de costos.

\section{INTRODUÇÃO}

A atenção domiciliar (AD) subsidiada pelo Sistema Único de Saúde (SUS) se estabeleceu com intuito de diminuir a sobrecarga de leitos hospitalares assim como seu alto custo, reduzir riscos de infecção e melhorar a qualidade de vida do paciente que, mesmo estando em condições de alta hospitalar, necessita de cuidados intensivos e recursos tecnológicos ${ }^{1}$. Adicionalmente, $\mathrm{AD}$ possibilita o convívio domiciliar e a participação da família que contribuem para melhora do paciente e retorno às atividades cotidianas ${ }^{1}$.
Nesse contexto de AD, a oxigenoterapia domiciliar prolongada (ODP) tem sido utilizada.

A prestação de ODP é um serviço de saúde que deve ser organizado e oferecido pelos municípios. Ressalta-se que a oferta de um serviço eficaz, que atenda às necessidades do usuário, é dever do Poder Público, que se justifica à luz da dignidade da pessoa ${ }^{2}$. A imposição de que tal serviço faça frente às necessidades do usuário está amparada nos princípios da integralidade e na resolutividade 2 . Para que seja possível a instalação de

IEnfermeira graduada pelo Centro Universitário Barão de Mauá. Ribeirão Preto, São Paulo, Brasil. E-mail: cristinashizuewatanabe@hotmail.com.

"Enfermeiro graduado pelo Centro Universitário Barão de Mauá. Ribeirão Preto, São Paulo, Brasil. E-mail: leonardoandradeef@hotmail.com.

IIIEnfermeiro graduado pelo Centro Universitário Barão de Mauá. Ribeirão Preto, São Paulo, Brasil. E-mail: mqsneto@hotmail.com.

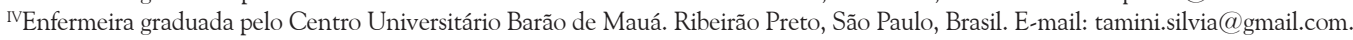

vEnfermeira. Doutora em Ciências. Professora Titular I do Curso de Graduação em Enfermagem do Centro Universitário Barão de Mauá. Enfermeira da

Prefeitura Municipal de Ribeirão Preto. São Paulo, Brasil. E-mail: 1suemi@hotmail.com. 
um serviço com eficácia que possibilite a implementação dos princípios referidos, faz-se necessária a identificação do perfil dos usuários de ODP.

Considerando o dever dos municípios no fornecimento dessa terapia, ressalta-se a necessidade do levantamento dos gastos financeiros. A gestão de custo constitui-se em componente importante dos sistemas de informações, já que pode ajudar a fornecer aos administradores melhores informações para a tomada de decisão. É útil para qualquer tipo de organização e proporciona uma visão mais acurada da realidade, pois permite olhar como são gastos os recursos disponíveis ${ }^{3}$.

Diante do exposto, essa pesquisa apresentou as seguintes questões norteadoras: qual o perfil dos usuários de ODP do Distrito Norte de Ribeirão Preto? Qual o custo de um mês da ODP desses usuários para o município?

Este estudo teve por objetivos identificar e analisar o perfil dos usuários de ODP, demonstrando o custo mensal da ODP de tais usuários para o município de Ribeirão Preto-São Paulo, Brasil.

\section{REVISÃo DE LITERATURA}

A oxigenoterapia domiciliar requer indicações que incluem pressão parcial de oxigênio no sangue arterial $(\mathrm{PaO} 2) \leq 55 \mathrm{mmHg}$ ou uma saturação de oxigênio arterial $(\mathrm{SaO} 2)$ de $88 \%$ ou menor no ar ambiente em repouso, ou com exercício ${ }^{4}$.

A ODP consiste em um procedimento de alto custo e a seleção dos beneficiados deve ser extremamente rigorosa e seguir recomendações internacionais, sendo a prescrição de uso bem detalhada quanto à fonte de oxigênio a ser utilizada, ao método de fornecimento, ao tempo de utilização e aos fluxos ${ }^{5}$. É considerado o principal tratamento não farmacológico para portadores de doença pulmonar obstrutiva crônica (DPOC) e hipoxemia crônica, tendo como objetivos principais: reversão de alterações hipoxêmicas, manutenção da hemoglobina, débito cardíaco e perfusão tecidual adequado ${ }^{6}$.

O uso de ODP por pelo menos 15 horas diárias apresenta impacto positivo com benefícios na hemodinâmica pulmonar, parâmetros hematológicos, capacidade de exercício, mecanismo pulmonar e estado mental. É uma das poucas terapias que mudam o prognóstico do paciente, já que vem a reverter a policitemia secundária, a hipoxemia, hipertensão arterial pulmonar, possíveis arritmias cardíacas, dispneias, melhorando a função neuromuscular e neuropsíquica e aumentando a tolerância a atividades físicas ${ }^{5-7}$.

Ainda com relação aos benefícios da terapia, estudos foram desenvolvidos indicando: evolução satisfatória da maioria dos pacientes em uso de ODP8, diminuição na utilização dos serviços de urgência por pacientes hipoxêmicos após início da terapia ${ }^{9}$, melhora na qualidade de vida de pacientes com DPOC e hipoxemia crônica ${ }^{10}$.
Destaca-se que a ODP tem sido pesquisada em populações de diferentes faixas etárias. Estudo com crianças ${ }^{11}$ mostrou que as doenças de base que levam ao uso de ODP são: fibrose cística, displasia broncopulmonar, bronqueolite obliterante, neurodeficiências e hipertensão pulmonar secundária. Já em adultos, pesquisa $^{12}$ considera que as principais doenças de base são: enfisema pulmonar, hipoxemia, bronquite, fibrose pulmonar entre outras; enquanto outro estudo ${ }^{13}$ cita a DPOC e hipoxemia. Quanto à ODP em diferentes grupos etários, salienta-se também que crianças apresentam particularidades relevantes, sendo necessário considerar, por exemplo, o crescimento físico e o desenvolvimento neurológico, além da necessidade de supervisão de um adulto e fornecimento da ODP na escola ${ }^{14}$.

Enfim a literatura encontrada indica o reduzido quantitativo de pesquisas relacionadas ao tema ODP $\mathrm{P}^{5-10,12-15}$. Os aspectos mais abordados são aqueles relacionados à patologia de base (que leva o paciente receber tal tratamento) e à importância de se utilizar critérios rigorosos para sua prescrição. Pode-se afirmar que investigações relacionadas ao uso de ODP têm sido desenvolvidas em diversas regiões do Brasil e do mundo, indicando levantamento de situações locais assim como divulgação de experiências.

\section{Metodologia}

Este estudo exploratório, descritivo e documental, na abordagem quantitativa, teve como campo de pesquisa Ribeirão Preto, município localizado no nordeste do Estado de São Paulo, Brasil, a 313 km da capital, com população de 612.339 habitantes ${ }^{16}$.

Ribeirão Preto se caracteriza como pólo regional de saúde, apresentando 15 unidades hospitalares. Sua rede municipal de atenção à saúde está estruturada em cinco distritos (leste, oeste, norte, sul e central), cada qual contendo uma unidade básica e distrital de saúde com atendimento 24 horas. Possui 46 unidades básicas de saúde, com 21 equipes de saúde da família ${ }^{17}$. O município também oferece um serviço de atenção domiciliar (SAD), implantado oficialmente em 1996.

O SAD tem por objetivos capacitar os profissionais que desempenham a $\mathrm{AD}$, definir critérios de inclusão e alta dos pacientes em $\mathrm{AD}$, diminuir período de internação hospitalar e reinternação, otimizar leitos e reduzir custos, assistir e apoiar a família, incentivando o desenvolvimento da responsabilidade da família, implementar as ações de AD, buscando a melhoria da qualidade de vida ${ }^{16}$. Conta com uma equipe multidisciplinar composta por: uma enfermeira coordenadora, três enfermeiras, dois técnicos de enfermagem e 10 auxiliares de enfermagem, quatro estagiários de enfermagem, um fisioterapeuta, três médicos, um dentista, sete aprimorandos, dois auxiliares administrativos e 
oito motoristas. Para cada Distrito de Saúde, há uma equipe de SAD implantada, que realiza o seguimento dos pacientes em uso de ODP ${ }^{18}$.

Ressalta-se que o cenário da pesquisa foi o SAD Norte, uma vez que o Distrito Norte, destinado à atuação administrativa e à prestação de serviços da instituição, foi o local de intervenção dos pesquisadores que desenvolveram a presente investigação.

Para coleta de dados, realizada no mês de julho de 2012, foram utilizados dados secundários captados nos prontuários dos usuários de ODP, arquivados na sede do SAD. Foi elaborado e utilizado um instrumento de coleta, contendo as variáveis: data de nascimento, sexo, idade de início da ODP, vinculação a plano de saúde; patologia de base, tabagismo; saturação oxigênio no início da ODP e durante a ODP, PaO2, tipo ou fonte de suporte oxigênio $(\mathrm{O} 2)$ e tempo de uso.

Para a organização dos dados coletados, foi elaborado um banco de dados no Programa Excel, levando-se em conta as variáveis utilizadas no instrumento de coleta.

Considerando os prontuários examinados, a população alvo foi constituída pelos 40 usuários de ODP cadastrados no SAD do Distrito Norte, sem exclusão.

Foram seguidas as normatizações da Comissão Nacional de Ética em Pesquisa (CONEP) traduzidas na Resolução no 196 do Conselho Nacional de Saúde (CNS). O projeto de pesquisa foi submetido ao Comitê de Ética em Pesquisa do Centro Universitário Barão de Mauá, através da Plataforma Brasil, sendo aprovado com o protocolo número 33992.

Para a apresentação e análise dos dados referentes ao perfil dos usuários de ODP, foi utilizada a estatística descritiva.

Em relação aos custos, para os usuários de cilindro, foi calculada a quantidade total de litros de $\mathrm{O} 2$ consumidos por mês, para cada cliente, mediante a fórmula: total de litros/mês $=(\mathrm{n} \times 60) \times \mathrm{h} \times 30$, sendo $\mathrm{n}=$ quantidade de litros/minuto de $\mathrm{O} 2 \mathrm{e} \mathrm{h}=$ quantidade hora de uso de O2. A partir daí, foi calculado o número de cilindros utilizados em um mês e posteriormente multiplicados esse número pelo valor do cilindro. Para usuários de ODP com concentradores, calculou-se o valor de locação mensal dos aparelhos e para usuários de ODP misto (cilindro e concentrador) foram realizados os dois cálculos descritos.

\section{Resultados e Discussão}

Ao analisar a idade dos 40 usuários de ODP, cadastrados no SAD do Distrito Norte, identificouse que houve variação de 3 a 91 anos (média: 61,4, mediana: 64 , desvio padrão: 20,1$)$, sendo que $3(7,5 \%)$ dos usuários tinham entre 3 a 19 anos e 27(67,5\%), estavam na faixa de 60 anos ou mais. Tais dados cor- roboram outro estudo que constatou serem os idosos a população que, grande parte das vezes, utiliza a ODP2. Ressalta-se que com a progressão da idade, algumas alterações fisiológicas tornam-se mais aparentes e a capacidade funcional do idoso pode estar ou ficar afetada, exigindo cuidados constantes ${ }^{19}$.

Observou-se também que 10(25\%) usuários tinham de 20 a 59 anos, representando uma população que se esperaria ser economicamente ativa. $\mathrm{O}$ uso da ODP limita o desenvolvimento de algumas atividades profissionais, podendo gerar perda de produtividade, indicando que os impactos das condições crônicas vão muito além dos gastos relacionados com a saúde ${ }^{20}$.

Em relação ao gênero, $27(67,5 \%)$ usuários eram do sexo feminino e $13(32,5 \%)$, do masculino. Ao observar a faixa etária e o gênero predominantes, pode-se estabelecer uma relação com expectativa de vida da população do Brasil. De acordo com pesquisa desenvolvida sobre a expectativa de vida dos brasileiros, mulheres vivem em média 77,32 anos e homens, 69,73 anos $^{21}$. Como os idosos foram o grupo que se destacou entre os usuários de ODP, pode se esperar uma maior porcentagem de mulheres que fazem uso dessa terapia, já que a expectativa de vida no sexo feminino é maior que no masculino.

No que se refere ao tabagismo, 12(30\%) dos usuários eram fumantes, segundo a Tabela 1 . Do total de 28 não fumantes, 14(50\%) foram tabagistas em outro momento da vida. É importante salientar que o tabagismo se constitui em uma ameaça à saúde e suas consequências negativas são reconhecidas há mais de quatro décadas ${ }^{20}$. Além disso, estudo realizado nos Estados Unidos evidencia o problema perene de queimadura secundária ao tabagismo em usuários de ODP22.

TABELA 1: Distribuição de usuários de ODP cadastrados no SAD na região do Distrito Norte de Ribeirão Preto, segundo gênero e uso de tabaco. Ribeirão Preto-SP, julho de 2012.

\begin{tabular}{lcccc}
\hline \multicolumn{1}{c}{$\begin{array}{c}\text { Tabagista } \\
\text { Sexo }\end{array}$} & f & \% & f & \% \\
\hline Masculino & 4 & 10 & 9 & 22,5 \\
Feminino & 8 & 20 & 19 & 47,5 \\
Total & 12 & 30 & 28 & 70 \\
\hline
\end{tabular}

Quando se analisa gênero e uso de tabaco, identifica-se que os dados encontrados e visualizados na Tabela 1 diferem dos resultados de pesquisa sobre a situação do tabagismo no Brasil ${ }^{23}$, que mostrou, de 2002 a 2009, a predominância do sexo masculino entre os fumantes em todas as regiões do país. Assim, destaca-se a importância dos profissionais desenvolverem ações (de pesquisas, prevenção e promoção de saúde) conscientizando o fumante para a busca de terapêutica antitabagismo ${ }^{24}$. 
Quanto à assistência dos serviços de saúde, 8(20\%) usuários são vinculados a plano de saúde. Esses dados mostram que, apesar de parte daqueles que utilizam a ODP possuir convênio, esses contratos não têm garantido o fornecimento da terapêutica, fazendo com que o município seja responsável pela oferta e garantia da mesma, o que pode ser explicado pelo fato de os convênios migrarem o paciente após alta hospitalar para continuidade do tratamento de ODP para o SUS, diminuindo seus gastos. Essa parcela de usuários de ODP com convênio pode diferir demasiadamente de outros distritos de saúde, já que o Distrito Norte se caracteriza por abranger uma área com população de baixas condições socioeconômicas e a cobertura do Sistema de Saúde Suplementar varia por classes de rendimentos familiares:

[...] na classe de menos de 1 salário mínimo sua cobertura é de 2,9\%; na classe de 1 a 2 salários mínimos, 6,7\%; na classe de 2 a 3 salários mínimos, 14,1\%; na classe de 3 a 5 salários mínimos, 24,9\%; na classe de 5 a 10 salários mínimos, 43,5\%; na classe de 10 a 20 salários mínimos, 65,8\%; e na classe de mais de 20 salários mínimos, de 83,9\% 25:54.

Ao se observar o perfil clínico dos usuários, identificou-se que 25(62,5\%) apresentaram DPOC como patologia de base, corroborando outro estudo que também identificou essa doença como a principal causa de ODP ${ }^{13}$.

Diante disso, reitera-se que a ODP é atualmente o principal tratamento não farmacológico para portadores de DPOC e hipoxemia crônica ${ }^{6}$. Ainda, estudo sobre qualidade de vida de pacientes com doença obstrutiva crônica de vias aéreas concluiu que tais usuários têm prejuízos na qualidade de vida que pode ser melhorada com o uso regular de ODP10.

Ainda com relação ao total de 25 (100\%) usuários de ODP com DPOC, constatou-se que 10(40\%) eram tabagistas, mesmo em uso da terapia, e 11(44\%) já haviam feito uso do tabaco em algum momento da vida. Diante desses resultados, salienta-se que o tabagismo, principal causa de DPOC, tem impacto multifatorial na sociedade, principalmente medido pela alta taxa de mortalidade.

Quando se analisa o tempo da doença, o mesmo variou de 2 meses a 31 anos. A maioria, 22 (55\%) registrou até 10 anos de tempo de diagnóstico conforme pode ser observado na Tabela 2.

TABELA 2: Distribuição dos usuários de ODP cadastrados no SAD do Distrito Norte de Ribeirão Preto, segundo tempo da doença. Ribeirão Preto-SP, julho de 2012.

\begin{tabular}{lcc}
\hline \multicolumn{1}{c}{ Tempo da doença } & $\mathbf{f}$ & \% \\
\hline$\leq 10$ anos & 22 & 55 \\
11 a 21 anos & 6 & 15 \\
22 a 32 anos & 1 & 2,5 \\
Sem informação & 11 & 27,5 \\
Total & 40 & 100 \\
\hline
\end{tabular}

Não foram localizados artigos que discorressem sobre a variável tempo da doença, o que pode estar relacionado ao fato deste atributo ser individualizado, de acordo com o biotipo, quadro clínico do paciente e a severidade da doença.

Em uma análise detalhada sobre o tempo da doença, observou-se que, apesar da DPOC ser a patologia mais frequente, esta doença apareceu com uma média de 8 anos de diagnóstico. Destaca-se que os pacientes que revelaram maior tempo da doença foram os que apresentaram bronquite asmática (20 anos) e fibrose cística (31 anos).

Em 11 (27,5\%) prontuários não havia dados inerentes ao tempo da doença, o que evidencia a necessidade de se verificar os motivos que estão levando à ausência de dados que deveriam contribuir para a identificação do histórico e das condições clínicas dos usuários.

Quanto ao tempo de uso de ODP, o mesmo variou de 2 meses a 22 anos (com média de 2,34 anos e mediana de 1,10 anos). Os resultados mostram que, no último ano, houve uma grande expansão de ODP na população estudada, já que 28(70\%) usuários cadastrados utilizavam ODP em tempo igual ou inferior há um ano. Ressalta-se que 7(17,5\%) usuários faziam uso da ODP há 2 ou 3 anos, conforme se observa na Figura 1, o que pode indicar aumento do acesso à terapia.

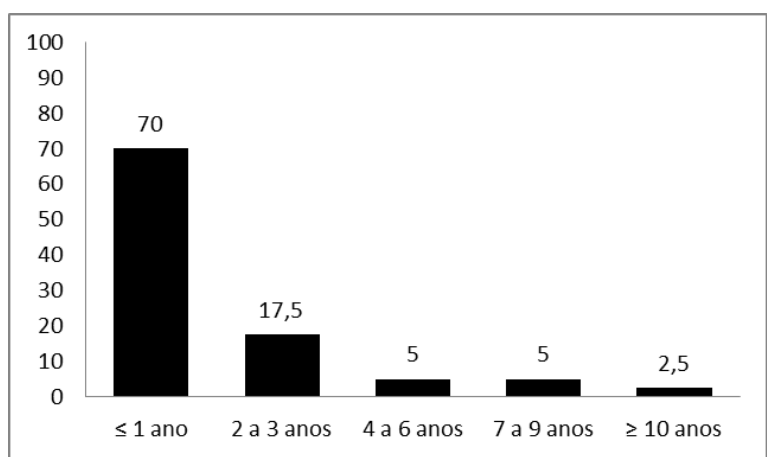

FIGURA 1: Distribuição percentual de usuários de ODP cadastrados no SAD na região do Distrito Norte de Ribeirão Preto, segundo o tempo de uso da ODP em anos. RibeirãoPreto-SP, julho de 2012.

Quando observado o nível de saturação inicial, no tratamento, a maioria dos usuários 30(75\%) apresentaram saturação menor que $88 \%$, o que justificava a necessidade da ODP. Durante o tratamento, 22(55\%) pacientes apresentaram saturação $\geq 90 \%$, o que mostra a eficiência da oxigenoterapia. Os dados vêm a corroborar outra pesquisa $a^{5}$ que informou ser esperada na ODP a obtenção de um fluxo de oxigênio maior ou igual 90\% em pacientes com hipoxemias crônicas 5 .

Em relação aos resultados referentes à $\mathrm{PaO} 2$, 25(62,5\%) usuários apresentavam $\mathrm{PaO} 2 \leq 55 \mathrm{mmHg}$, mostrando que os valores $\mathrm{PaO} 2$ como indicação para 
início do ODP estão de acordo com os citados na literatura: pacientes que devem fazer uso da terapia de ODP são aqueles que apresentam $\mathrm{PaO} 2<55 \mathrm{mmHg}$ ou $\mathrm{SaO} 2<88 \%$ com ou sem hipercapnia ${ }^{26}$. Salientase que aqueles que apresentam $\mathrm{PaO} 2$ entre $55 \mathrm{mmHg}$ e $60 \mathrm{mmHg}$ ou $\mathrm{SaO} 2$ de $88 \%$ podem ter indicação de ODP caso revelem evidências de hipertensão arterial pulmonar, edema periférico sugestivo de insuficiência cardíaca congestiva ou policitemia ${ }^{5}$.

Quanto à fonte de suporte de $\mathrm{O} 2,3(7,5 \%)$ usuários utilizavam fonte mista, $17(42,5 \%)$ usavam o cilindro e 20(50\%), o concentrador. Esses dados podem estar relacionados ao custo, pois pesquisadores mencionam que os cilindros se esvaziam rapidamente e necessitam ser substituídos por outro constantemente, o que encarece a terapia, diferente do concentrador que utiliza o ar ambiente como meio de captar o $\mathrm{O} 2^{5}$. Outro fator, neste caso, que define a fonte é a renda familiar e a estrutura do domicílio, pois o concentrador exige consumo de energia elétrica. Adicionalmente, em locais em que há queda contínua de energia, usuários de ODP não podem ficar sem cilindro no domicílio ${ }^{27}$.

Em relação à via de administração de $\mathrm{O} 2$, 35(87,5\%) usuários utilizavam cateter nasal e 5(12,5\%) usavam outras vias como: máscara facial, máscara traqueal. Cada tipo de via de administração é indicado conforme o grau de comprometimento do aparelho respiratório do paciente. Outro estudo também revela que a maioria dos usuários utilizava a via de administração por cateter, indicando que os mesmos não necessitavam de grandes volumes de $\mathrm{O} 2$, já que o cateter de $\mathrm{O} 2$ suporta o fluxo máximo de 6 litros/minuto ${ }^{4}$.

Referente ao fluxo de $\mathrm{O} 2$, a dose de $\mathrm{O} 2$ administrada deve ser indicada individualmente, conforme o fluxo necessário para se obter um valor de $\mathrm{PaO} 2$ de pelo menos $60 \mathrm{mmHg}$ e uma $\mathrm{SaO} 2$ maior que $90 \%$ em repouso ${ }^{28}$. $\mathrm{Na}$ presente investigação, 18(45\%) mantiveram o fluxo de $\mathrm{O} 2$ (apresentando quadro clínico estável, sem avanços), 13(32,5\%) precisaram de aumento de fluxo de $\mathrm{O} 2$ (indicando piora na situação clínica) e 9(22,5\%) diminuíram o fluxo de $\mathrm{O} 2$.

A análise do perfil clínico dos usuários de ODP mostrou que os mesmos estão dentro dos critérios de inclusão de ODP do SAD e em conformidade com padrões estabelecidos em revisões literárias ${ }^{26-28}$.

Ao se iniciar a identificação dos custos da ODP, salienta-se que, no momento da pesquisa, a empresa fornecedora de cilindro cobrava $\mathrm{R} \$ 110,00$ por cilindro de 10 metros cúbicos $(10 \mathrm{~m} 3)$ - tamanho utilizado em ODP no município e R $\$ 160,00$ pelo aluguel de cada concentrador.

Identificou-se que 20 (50\%) usuários de ODP utilizam o concentrador. Multiplicando esse total de usuários (20) de concentrador pelo valor do aluguel do aparelho por mês $(\mathrm{R} \$ 160,00)$, obtém-se um custo $\mathrm{R} \$ 3.200,00$.
Quanto à fonte de $\mathrm{O} 2$, observou-se que 17 $(42,5 \%)$ usuários faziam uso somente de cilindro. Cabe reiterar que, para cada um deles, foi utilizada a fórmula descrita na metodologia para calcular a quantidade total de litros de $\mathrm{O} 2$ consumidos em um mês.

Esses 17(42,5\%) usuários de cilindro utilizam um total 140.000 litros de $\mathrm{O} 2$ por mês. Este valor dividido por 10.000 (que equivale a um cilindro de $10 \mathrm{~m}^{3}$ ) resulta em um total de 140 cilindros de $10 \mathrm{~m}^{3}$ por mês. Ao se multiplicar o número de cilindros (140) pelo valor de cada cilindro de $10 \mathrm{~m}^{3}(\mathrm{R} \$ 110,00)$, alcança-se um custo de $\mathrm{R} \$ 15.400$.

A pesquisa identificou 3 (7,5\%) usuários que utilizam fonte mista de $\mathrm{O} 2$, ou seja, concentrador e cilindro. Multiplicando 3 (número de usuários) por $\mathrm{R} \$ 160,00$ (valor do aluguel do concentrador por mês), tem-se um custo de $\mathrm{R} \$ 480,00$. Para esses 3 (7,5\%) usuários foi realizado o cálculo, multiplicando $R \$ 110,00$ (valor do cilindro) por 3 (número de usuários), obtendo um custo de $\mathrm{R} \$ 330,00$. Ressalta-se que não foi possível o acesso ao modo que estes pacientes utilizam as fontes mistas, portanto, considerou-se para esses usuários, o fornecimento de pelo menos um cilindro/mês. Somando os valores dos gastos com cilindro $(\mathrm{R} \$ 330,00)$ e concentradores $(\mathrm{R} \$ 480,00)$, obtém-se um custo total de $\mathrm{R} \$ 810,00$ para os usuários em ODP com fonte mista.

Os custos identificados mostram que, no cenário desta pesquisa, o cilindro é mais oneroso do que o concentrador, o que corrobora outro estudo, no qual o cilindro teve um custo mais alto do que o concentrador $^{21}$. Cabe ressaltar que valores de energia elétrica para utilização de concentrador não foram calculados.

Somando todos os gastos citados ( $\mathrm{R} \$ 3.200,00$ $+\mathrm{R} \$ 15.400,00+\mathrm{R} \$ 810,00)$, no total, o município apresentou, no mês estudado, uma despesa com ODP de $\mathrm{R} \$ 19.410,00$.

Ao realizar a soma total de dias dos usuários de ODP em AD pesquisados, pode-se inferir que se evitaram 34.283 dias de internações hospitalares. Não foi possível obter o valor de uma diária de internação hospitalar, considerando que o cálculo da mesma depende de vários fatores (recursos disponibilizados como alimentação, equipe multiprofissional, entre outros).

Em um estudo, realizado com 40 crianças em uso de ODP, os autores informam que para os 16.984 dias de hospitalização, estimou-se que o sistema de saúde gastaria cerca de $\mathrm{R} \$ 186.824,00$ com a permanência desses usuários no hospital. Nessa investigação, foi realizado um cálculo de custo, considerando o valor de $\mathrm{R} \$ 11,00$ por dia de internação ${ }^{8}$. Se for utilizado esse mesmo valor para se obter os custos evitados com internação hospitalar neste estudo, multiplicando $\mathrm{R} \$ 11,00$ por 34.283 dias, tem-se que a despesa de $\mathrm{R} \$ 377.113,00$ seria evitada com o uso da ODP no domicílio. Certamente, o valor real é bem superior a esse, uma vez que o estudo citado foi publicado em $2001^{8}$. 
Nesse momento, é importante considerar a limitação do presente estudo, já que não foi possível identificar o custo de uma diária de internação hospitalar, pois o mesmo varia de acordo com inúmeros fatores (setor, materiais, via de alimentação, entre outros).

Reitera-se que o cilindro foi responsável por $\mathrm{R} \$ 15.400,00$ (79\%) dos custos identificados com a terapia, evidenciando ser mais oneroso no Distrito. A substituição dos cilindros por concentradores poderá reduzir os custos significativamente para o município. Em contrapartida, ressalta-se que devem ser analisadas as questões sociais dos usuários antes de se pensar na possibilidade de mudança, devido ao alto consumo de energia elétrica gerada pelo concentrador.

\section{ConClusão}

O estudo trouxe resultados que expressam a realidade de uma região específica do município, possibilitando o conhecimento de um contexto local que pode auxiliar gestores e trabalhadores no desenvolvimento de ações no dia a dia do trabalho.

Conclui-se que o perfil dos usuários (predomínio de idosos, com doença crônica, em uso de tabaco em algum momento da vida) indica resultados que retratam um panorama vivenciado no país e no mundo: transição demográfica e mudança do perfil de morbidade, ou seja, envelhecimento da população e prevalência das doenças crônicas relacionadas ao estilo de vida, gerando demanda para tratamentos em longo prazo. Reitera-se, também, que o perfil clínico dos usuários segue os parâmetros de indicação da ODP do SAD e da literatura.

Finalizando, ao se considerar os custos e o número de dias de internação hospitalar evitados com o uso da ODP na AD, conclui-se que o estabelecimento dessa terapia favorece a diminuição de problemas enfrentados no sistema de regulação de vagas, destacando a importância do SAD de Ribeirão Preto na trama do SUS.

\section{REFERÊNCIAS}

1.Ministério da Saúde (Br). Lei no 10.424 de 15 de abril de 2002. Dispõe sobre as condições para a promoção, proteção e recuperação da saúde [...] regulamentando a assistência domiciliar no Sistema Único de Saúde. [citado em 14 abr 2014]. Disponível em: http://portal. saude.gov.br.

2.Oliveira LM. Responsabilidade municipal pela prestação do serviço de oxigenoterapia domiciliar e seus contornos. Rev Direito Sanitário. 2009; 10(1): 39-50. 3.Almeida AG, Borba JA, Flores LCS. A utilização das informações de custos na gestão da saúde pública: um estudo preliminar em secretarias municipais de saúde do Estado de Santa Catarina. Rev Administração Pública. 2009; 4: 579-607.
4.Perry A. Oxigenação. In: Potter PA, Perry A. Fundamentos de enfermagem. Rio de Janeiro: Elsevier; 2009. p.907-65. 5.Bartholo TP, Gomes MM, Noronha Filho JA. DPOC: o impacto da oxigenoterapia domiciliar no tratamento. Pulmão RJ - Atualizações Temáticas. 2009; 1 (1): 79-84. 6.Viegas CAA, Adde FV, Paschoal IA, Godoy I, Machado MCLO. Oxigenoterapia domiciliar prolongada (ODP). J bras pneumol. 2000; 26: 341-50.

7.Alves MV, Godoy I, Luppi CH. Levantamento das características dos pacientes atendidos no serviço de oxigenoterapia da Faculdade de Medicina de BotucatuUNESP. Rev Ciên Ext. 2004; 1 (1): 53-4.

8. Mocelin HT, Fisher GB, Ranzi LC, Rosa RD, Philomena MR. Oxigenoterapia domiciliar em crianças: relato de sete anos de experiência. J bras pneumol. 2001; 27: 148-52.

9.Munhoz AS, Adde FV, Nakaie CMA, Doria Filho U, Silva Filho LVRF, Rodrigues JC. Oxigenoterapia domiciliar prolongada em crianças e adolescentes: uma análise do uso clínico e de custos de um programa assistencial. J Pediatr. 2011; 87 (1): 13-8.

10.Tanni ES, Vale SA, Lopes PS, Guiotoko MM, Godoy I, Godoy I. Influência do sistema de fornecimento de oxigênio na qualidade de vida de pacientes com hipoxemia crônica. J bras pneumol. 2007; 33: 161-7.

11.Balfour-Lynn IM. Oxigenoterapia domiciliar prolongada: uma perspectiva britânica. J Pediatr. 2011; 87 (1): 1-3. 12.Baldi BG, Pereira CAC, Rubin AS, Santana, ANC, Costa AN, Carvalho, CRR et al. Destaques das Diretrizes de Doenças Pulmonares Intersticiais da Sociedade Brasileira de Pneumologia e Tisiologia. J bras pneumol. 2012; 38: 282-91.

13. Nocturnal Oxygen Therapy Trial Group. Continuous or nocturnal oxygen therapy in hypoxemic chronic obstructive lung disease: a clinical trial. Annals of Internal Medicine. 1980; 93: 391-8.

14.Adde FV, Alvarez AE, Barbisan BN, Guimaraes BR. Recommendations for long-term home oxygen therapy in children and adolescents. J Pediatr. 2013;89(1):6-17 15. Comité Nacional de Neumonología. Guías para el manejo de la oxigenoterapia domiciliaria en pediatría. Arch Argent Pediatr. 2013;111:448-54.

16.Instituto Brasileiro de Geografia e Estatística. IBGE. Estimativa populacional 2011. [citado em 14 abr 2014]. Disponível em: http://ibge.gov.br//.

17.Ribeirão Preto (SP). Secretaria Municipal da Saúde. Divisão de Planejamento em Saúde. Plano Municipal de Saúde 2010-2013. Ribeirão Preto (SP): Secretaria Municipal da Saúde; 2009.

18.Ribeirão Preto (SP). Secretaria Municipal da Saúde. Protocolo de atendimento em oxigenoterapia domiciliar prolongada (ODP). Ribeirão Preto (SP): Secretaria Municipal da Saúde; 2007.

19.Martins JJ, Nascimento ERP, Erdmann AL, Candemil MC, Belaver GM. O cuidado no contexto domiciliar: o discurso de idosos/familiares e profissionais. Rev enferm UERJ. 2009; 17:556-62.

20. Organização Mundial da Saúde. Cuidados inovadores para condições crônicas de saúde: componentes estruturais de ação: relatório mundial. Brasília (DF): Organização Mundial da Saúde; 2003. 
21.Instituto Brasileiro de Geografia e Estatística. Tabuas completas de mortalidade 2010. [citado em 14 abr 2014]. Disponível em http://www.ibge.gov.br

22 Al Kassis S, Savetamal A, Assi R, Crombie RE, Ali R; Moores C, Najjar A, Hansen T, Ku T, Schulz JT. Characteristics of patients with injury secondary to smoking on home oxygen therapy transferred intubated to a burn center. J Am Coll Surg. 2014; 218:1182-6.

23. Ministério da Saúde (Br). Instituto Nacional de Câncer INCA. A situação do tabagismo no Brasil: dados do inquérito do sistema internacional de Vigilância, da Organização Mundial da Saúde, realizado no Brasil, entre 2002 e 2009. Rio de Janeiro: INCA; 2011.

24.Scherer EA, Scherer ZAP. Opinião de pacientes, familiares e profissionais sobre o hábito de fumar em uma enfermaria psiquiátrica. Rev enferm UERJ. 2014; 22:376-82.
25.Conselho Nacional de Secretários da Saúde. SUS: avanços e desafios. Brasília (DF): CONASS; 2006.

26.Global strategy for the diagnosis, management, and prevention of chronic obstructive pulmonary disease. Global Initiative for Chronic Obstructive Lung Disease (GOLD), executive summary, 2006. [cited in 2014 Apr 14]. Disponível em: http//goldcopd.com

27.Sato K, Morita R, Tsukamoto K, Sato N, Sasaki Y, Asano M, Okuda Y, Miura H, Sano M; Kosaka T, Watanabe H, Shioya T, Ito H. Questionnaire survey on the continuity of home oxygen therapy after a disaster with power outages. Respir Investig. 2013; 51(1):9-16.

28.Cedano S, Belasco AGS, Traldi F, Machado MCLO, Bettencourt ARC. Influência das características sociodemográficas e clínicas e do nível de dependência na qualidade de vida de pacientes com DPOC em oxigenoterapia domiciliar prolongada. J bras pneumol. 2012; 38: 331-8. 